\section{Ökologisches Benchmarking - ein Phantom?}

\author{
Welche Ansäłze des ökologischen Vergleichs mittels Kennzahlen sind in \\ Deutschland vorhanden und wie lassen sie sich klassifizieren? In einer 1998 \\ vom IÖW durchgeführten Vorstudie wurden dazu 30 Unternehmensverbände \\ befragt. Mit nur wenigen Ausnahmen ergibt sich ein diffuses Bild von wenig \\ koordinierten Aktivitäten.
}

$\mathrm{D}$ Von Steffen Lange und Heinz Kottmann marking als einen unternehmensinternen oder -ïbergreifenden Vergleichsprozeß von Kennzahlen mit festgelegten Richtwerten oder Zielgrößen als Instrument zur Ableitung von Verbesserungsmaßnahmen und -zielen. Ökologisches Benchmarking bezieht sich auf Umweltkennzahlen und Umweltziele. Es unterstiitzt ein umfassendes ökologisches Lernen von Unternehmen und Branchen, allerdings steht dieses Instrument erst am Anfang. Es lohnt daher, alle bestehenden Ansätze eines ökologischen Benchmarking - seien es unternehmens- oder konzerninterne Benchmarks, Branchen-Benchmarks oder Initiativen des ökologischen Ratings - für eine Weiterentwicklung zu nutzen.

Das Fazit aus der Befragung stellt sich aber recht bescheiden dar (1). Die vielen Gespräche und Interviews mit den Experten der großen deutschen Verbände haben gezeigt, daß dieses Thema für die einen seiner Zeit noch etwas weit voraus gegriffen ist, für die anderen ein längst alltägliches Mittel im Umgang mit den Umweltproblemen der Unternehmen ist. Einige Dienstleister, insbesondere die Banken, haben offenbar weniger Berührungsängste bezüglich der Darstellung ihrer Umweltauswirkungen durch ihre Produkte und Tätigkeiten. Manche Dienstleister weisen gemessen an den Mitarbeitern höhere Strom- oder Wasserverbräuche als Unternehmen des produzierenden Gewerbes auf (2). Aber auch die Branchenverbände, denen eine hohe Umweltbelastung zugeschrieben wird, wie die Chemische Industrie (VCI), die Automobilhersteller (VDA) oder die Maschinen- und Anlagenbauer (VDMA), treten dem ökologischen Benchmarking aufgeschlossen gegenüber und bieten teils eigene Aktivitäten an.
Besonders positiv ist der Verein für Umweltmanagement in Banken, Sparkassen und Versicherungen e.V. (VfU) hervorzuheben, der als Vorreiter auf dem Gebiet der Kennzahlenbildung mit Umweltrelevanz und deren Vergleich innerhalb der Branche anzusehen ist. So war auch er der erste, der seinen Mitgliedern Richtlinien zur umweltgerechten Bilanzierung und einen Leitfaden zur Umweltberichterstattung an die Hand gegeben hat.

Allerdings zeigt Abbildung 1, daß nicht einmal die Hälfte der befragten Verbände ein konventionelles und nur fünf ein ökologisches Benchmarking anbieten. Problematisch erweist sich dabei die Tatsache, daß die Weiterentwicklung dieses Instrumentes an vielen Verbänden ,vorïberzieht": Dies geht darauf zurück, daß bei ihnen die Offenheit für den Vergleich von umweltrelevanten Daten vielfach noch nicht gegeben ist. Es bestehen vor allem Befürchtungen einer Verschärfung des Wettbewerbs.

\section{Vielfältige Hemmnisse}

Ökologisches Benchmarking scheitert dabei zum einen an der oft noch unzureichenden Organisa-

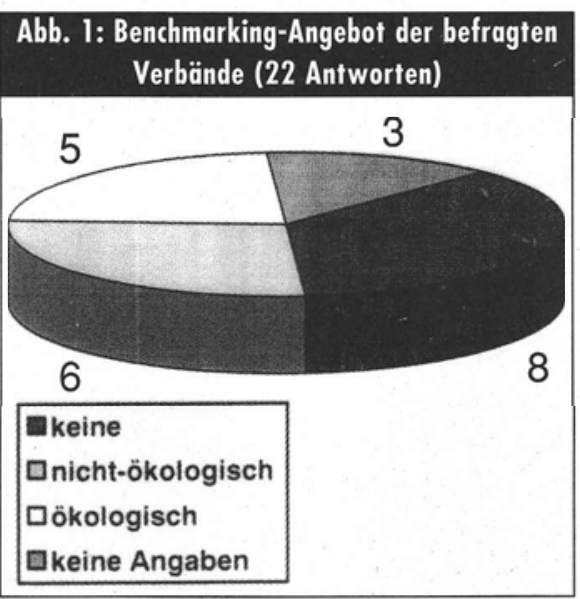

Quelle eigene Darstellung tion der Unternehmen in Umweltbelangen und zum anderen an der mangelnden Kommunikationsbereitschaft. Gerade kleinen Unternehmen fällt es schwer, sich mit Unternehmen, die ähnliche Interessen im Umweltschutz verfolgen, abzustimmen und $\mathrm{zu}$ vergleichen. Es überwiegen oftmals andere Interessen und die Scheu vor der Suche nach geeigneten Vergleichspartnern. Um so schwieriger ist dies, wenn der Branchenverband in diesen Bereichen keine Hilfestellungen gibt. Dabei fällt die Frage nach dem weiteren Bedarf der Verbandsmitglieder nach Benchmarkingaktivitäten sehr unterschiedlich aus. Immerhin 7 Verbände haben häufige Anfragen, während der Großteil keinen Überblick hat oder keine Angaben macht. Der Öffentlichkeitsbezug wird eher restriktiv gesehen. Nur vereinzelt machen die Verbände ihre im Benchmarking gesammelten Umweltdaten der Öffentlichkeit direkt zugänglich.So legt der VCI großen Wert auf die Öffentlichkeitsarbeit, vermeidet aber die Darstellung von einzelnen Unternehmensdaten und präsentiert die Ergebnisse als Branchendaten. Der VDMA führt die weitestgehenden Erhebungen zum Umweltschutz durch. Die Veröffentlichung der Daten obliegt aber den einzelnen VDMA-Mitgliedern. So ist es nicht verwunderlich, daß viele verdeckte Ansätze existieren, dennoch zur Zeit aber keine Struktur zu erkennen ist. Ökologisches Benchmarking wird daher wohl noch einige Zeit ein Thema unter vorgehaltener Hand bleiben, solange sich die ersten Versuche der Unternehmen und Verbände im Verborgenen abspielen. Zwar ist konzeptionell noch einiges an Arbeiten zu leisten, doch bestärken die wenigen Beispiele die Vermutung, daß ökologisches Benchmarking große Potentiale bietet.

\section{Anmerkungen}

(1) Steffen Lange: Ökologisches Benchmarking. Theoretische Grundlagen und Stand der Praxis. Unveröffentlichte Vorstudie.

(2) Rouberger, Rainer: Renchmarking mit Umweltkennzahlen bei Banken. In: Heinz Kottmann, Jens Clousen (Hrsg.): Ökologisches Benchmarking von Unternehmen. IÖW-Schriftenreihe 133/98.

\section{Die Autoren}

Steffen Lange ist Diplom-Betriebswirt der FHTW Berlin, Heinz Kottmann ist wissenschaftlicher Mitarbeiter am IÖW.

Kontakt: Heinz Kottmann, IÖW, Giesebrechtstr.13, 10629 Berlin. Tel. 030/ 884594-0, Fax 030/ 8825439, E-mail: Heinz.Kottmann@ioew.de 
(c) 20I0 Authors; licensee IÖW and oekom verlag. This is an article distributed under the terms of the Creative Commons Attribution Non-Commercial No Derivates License (http://creativecommons.org/licenses/by-nc-nd/3.o/), which permits unrestricted use, distribution, and reproduction in any medium, provided the original work is properly cited. 